\title{
Distributionally Robust Generation Expansion Planning Model Considering RES Integrations
}

\section{Conference Paper}

\section{Author(s):}

Han, Xuejiao; Hug, Gabriela

Publication date:

2019-10-24

\section{Permanent link:}

https://doi.org/10.3929/ethz-b-000348483

Rights / license:

In Copyright - Non-Commercial Use Permitted

Originally published in:

https://doi.org/10.1109/ISGT-Asia.2019.8881614 


\title{
Distributionally Robust Generation Expansion Planning Model Considering RES Integrations
}

\author{
Xuejiao Han, Gabriela Hug \\ Department of Information Technology and Electrical Engineering, ETH Zurich \\ Email: \{xuhan, hug\}@eeh.ee.ethz.ch
}

\begin{abstract}
A generation expansion planning strategy to derive the optimal generation mix for distribution systems is proposed in this paper. The considered candidate units include decentralized storage units, variable generation units, dispatchable generation units and flexible loads. The uncertainties in demand, wind and PV generation forecasts are characterized by an ambiguity set that defines a family of distributions. Following the principle of distributionally robust optimization (DRO), a two-stage stochastic programming model is formulated to minimize the worst-case expectation of the sum of long-term investment costs and short-term operational costs. A case study demonstrates the effectiveness of the proposed model and the importance of considering more statistical characteristics of uncertain variables. The impacts of forecast errors' variances are analyzed and the results of using DRO with that of using robust optimization and stochastic optimization are compared.
\end{abstract}

Index Terms-Decentralized energy resources, distribution system, distributionally robust optimization, generation expansion planning, linear decision rule.

\section{INTRODUCTION}

\section{A. Motivation and Goal}

In recent years, due to favorable financial subsidies and ambitious renewable energy targets, the electricity industry worldwide is experiencing a shift towards decentralization. The investments in decentralized energy resources (DERs) has the additional benefit that it reduces grid losses and possibly decreases or defers capacity investments. These effects are strengthened by the ongoing decrease in their investment costs. However, due to the variable and uncertain natures of their power outputs, DERs might face the challenge of high imbalance costs. In order to manage DERs in a proper way and to fully utilize the value of their investments, a generation expansion planning model based on DRO is proposed.

\section{B. Related Works and Contributions}

While a lot of work has been done in terms of generation expansion planning, most investment models mainly focus on deterministic problems or consider uncertainties using traditional stochastic optimization, e.g. [1]-[3]. However, the random parameters' distribution for stochastic optimization (SO) is rarely known and SO often suffers from high computational complexity resulting from a large number of scenarios. Robust optimization ( $\mathrm{RO}$ ) on the other hand does not require knowledge of the uncertainty distribution and is used to obtain decisions that optimize the worst-case scenario over an uncertainty set, e.g. [4], [5]. Its model is often computationally tractable as the uncertainty considered is distribution free and described only by an uncertainty set, which results in much lower computational cost but also more conservative results.

This work was supported by the Swiss Federal Office of Energy within SCCER FURIES and the Nexus project.
Different from $\mathrm{SO}$ and $\mathrm{RO}$, in distributionally robust optimization (DRO), the worst-case is taken over an ambiguity set, i.e. a family of distributions described by certain statistical characteristics (e.g. expectation and standard deviation) of the unknown data-generating distribution [6]. Recently, DRO has also been applied to power system problems [7], [8]. In [7], a unit commitment model considering uncertain wind power generation using DRO is proposed whereas in [8], a DRO model to solve the energy and reserve dispatch problem is presented. In the latter, it is modeled as a semi-definite programming problem and the delayed constraint generation algorithm was used to solve it in a tractable manner.

To our knowledge, however, the application of DRO to generation expansion planning (GEP) models is novel. With the growing penetration of DERs, it is increasingly important to build generation expansion planning models taking uncertainties into consideration. Consequently, the focus of this paper is on joint investment and operation optimization of DERs, considering the uncertainties of PV, wind and demand forecast errors using distributionally robust optimization. The contributions of this paper are:

1) To propose a two-stage stochastic programming model that jointly optimizes the investments and operations of a distribution system considering storage units, flexible load units, variable and dispatchable generation units.

2) To model uncertainties of forecast errors of variable generation outputs and demand using DRO.

3) To analyze effects of statistical information and compare the results of using DRO with that of using RO and SO.

The rest of the paper is organized as follows: the problem description is presented in Section II and the mathematical formulations and the proposed optimization model are given in Section III. Section IV presents the results of the case study, and finally conclusions are drawn in Section V.

\section{PROBLEM DESCRIPTION}

In this paper, questions concerning the optimal decentralized generation mix for a distribution system are addressed, considering uncertainties of variable generation units' outputs and demand forecasts. Similar to previous work [9], in order to compensate the imbalances caused by variable generation units, to enhance the efficiency of electricity utilization and to contribute to flexibility provisions, the characteristics of diverse units are aggregated into a single entity (i.e. aggregator). The aggregator, which can also be a distribution system operator, is modelled as a cluster of storage devices, flexible loads, dispatchable and variable generation units and a single dispatch portfolio is therefore generated.

The structure of the proposed two-stage optimization model is set up as follows: 
- 1st stage: The aggregator optimizes the investment decisions (i.e. investment capacity for each technology).

- 2nd stage: The aggregator decides its short-term operations considering various uncertainties.

As this is a multi-stage stochastic programming model, the non-anticipativity characteristic is taken into account to guarantee that if realizations of stochastic processes are identical up to stage $n$, then their decision variables are identical up to that stage [10]. The objective of the model is to minimize the total costs of all stages.

\section{Formulations OF OPTIMIZATION PROBLEM}

This section presents the derivation of the DRO, RO and SO formulations of the GEP problem. We start with a general stochastic formulation of the problem in Section III-A. However, the original form of the problem is intractable since the optimized solution is required to be feasible for all possible realizations of uncertainties. Therefore, in Section III-B, based on the principle of DRO, a tractable two-stage second-order cone (SOC) model is derived by approximating the second-stage operational decisions using linear decision rules (LDRs). An ambiguity set is constructed to describe a family of possible distributions of the random variables, using statistical information extracted from historical data. Formulations applying SO and RO are presented in Section III-C. Three different levels of uncertainties related to demand, wind and PV output forecasts are considered in this paper.

\section{A. Formulations of Stochastic Problem}

To optimize the investment and operation decisions, different groups of constraints need to be considered.

1) Investment Constraints: The capacity of resource $k \in \mathbf{K}$ to be invested in the distribution system is non-negative, where $K$ is the set of considered technologies, i.e.

$$
0 \leq x_{k}^{\text {inv }}, \quad \forall k \in \mathbf{K}
$$

2) Operation Constraints: The operational limits of the considered four different types of components in the planned distribution system, i.e. dispatchable and variable generation units, storage units and flexible loads, need to be modelled.

a) Dispatchable Generation Unit: The power output $P_{g, t}$ of dispatchable generation unit $g$ at time $t$ is non-negative and limited by its invested capacity $x_{g}^{\text {inv }}$. Mathematically,

$$
0 \leq P_{g, t} \leq x_{g}^{\text {inv }}
$$

b) Variable Generation Unit: Two types of variable generation technologies, namely wind and PV, are considered in this work. The power output of variable generation unit $v \in \mathbf{V}$ at time $t$ indicated by $P_{v, t}$ is non-negative and limited by the product of the invested capacity $x_{v}^{\text {inv }}$ and the sum of generation forecasts in percentage $p_{v, t}^{\mathrm{f}}$ and forecast error $\delta_{t}^{v}$, where $\boldsymbol{V}$ denotes the set of all variable generation units, i.e.

$$
0 \leq P_{v, t} \leq x_{v}^{\text {inv }}\left(p_{v, t}^{\mathrm{f}}+\delta_{t}^{v}\right), \quad \forall v \in \mathbf{V}
$$

The curtailment of these units is assumed to be free. c) Storage Unit: The energy $E_{s, t}$ stored in storage unit $s$ is non-negative and limited by the invested capacity $x_{s}^{\text {inv }}$. The end storage level $E_{s, T}$ is set to equal the initial level $E_{s, 0}$. The inflow $P_{s, t}^{\mathrm{ch}}$ and the outflow $P_{s, t}^{\text {dis }}$ of unit $s$ at time $t$ are limited by the maximum inflow $P_{s}^{\text {ch,max }}$ and the maximum outflow $P_{s}^{\text {dis,max }}$ defined as percentage of the capacity. Mathematically,

$$
\begin{aligned}
& 0 \leq E_{s, t} \leq x_{s}^{\text {inv }} \\
& 0 \leq P_{s, t}^{\text {ch }} \leq P_{s}^{\text {ch,max }} x_{s}^{\text {inv }} \\
& 0 \leq P_{s, t}^{\text {dis }} \leq P_{s}^{\text {dis,max }} x_{s}^{\text {inv }} \\
& P_{s, t}^{\text {ch }}-P_{s, t}^{\text {dis }} \leq P_{s}^{\text {ch,max }} x_{s}^{\text {inv }} \\
& P_{s, t}^{\text {dis }}-P_{s, t}^{\text {ch }} \leq P_{s}^{\text {dis,max }} x_{s}^{\text {inv }} \\
& E_{s, T}=E_{s, 0}
\end{aligned}
$$

Finally, the relationship of the storage levels for two consecutive time steps is defined by

$$
E_{s, t}=E_{s, t-1}+\eta_{s} P_{s, t}^{\mathrm{ch}}-\eta_{s}^{-1} P_{s, t}^{\mathrm{dis}}
$$

where $\eta_{s}$ is the conversion efficiency of storage unit $s$.

d) Load: It is assumed that a certain percentage of the load defined by $L^{\text {sh,max }}$ is flexible and can be shifted. Moreover, to lower the effects of the demand shifting on customers, the daily demand consumption is assumed to be constant, i.e.

$$
\begin{aligned}
& -L^{\mathrm{sh}, \max } P_{l, t}^{\mathrm{f}} \leq P_{l, t}^{\mathrm{sh}} \leq L^{\mathrm{sh}, \max } P_{l, t}^{\mathrm{f}} \\
& \sum_{t=1}^{24} P_{l, t}^{\mathrm{sh}}=0
\end{aligned}
$$

where $P_{l, t}^{\mathrm{f}}$ and $P_{l, t}^{\mathrm{sh}}$ are the demand forecast and the shifted demand at time $t$, respectively.

e) Power Balance Constraint: To guarantee the reliability and stability of a power system, supply and demand must be balanced at all times, i.e. for each time period $t$ the total demand minus the load shedding must equal the sum of the energy generated by dispatchable and variable units and drawn from the storage minus the energy used to charge the storage:

$$
P_{g, t}+\sum_{v \in \mathbf{V}} P_{v, t}+P_{s, t}^{\mathrm{dis}}-P_{s, t}^{\mathrm{ch}}=P_{l, t}^{\mathrm{f}}\left(1+\delta_{t}^{l}\right)+P_{l, t}^{\mathrm{sh}}-P_{l, t}^{\mathrm{shed}}
$$

where $\delta_{t}^{l}$ and $P_{l, t}^{\text {shed }}$ are the demand forecast error and the load shedding at time $t$.

f) Objective: The goal of the model is to optimize investment and operation decisions of decentralized units, so as to minimize the total costs defined by the sum of the firststage investment cost and the second-stage operating cost over the whole simulation period. Mathematically,

$$
\begin{aligned}
C^{\text {inv }} & =\sum_{k \in\{\mathbf{V}, g, s\}} \alpha_{k}^{\text {ann }} c_{k}^{\text {inv }} x_{k}^{\text {inv }} \\
C_{t}^{\text {op }} & =\sum_{k \in\{\mathbf{V}, g\}} c_{k}^{\text {op }} P_{k, t}+c_{s}^{\text {op }}\left(P_{s, t}^{\mathrm{ch}}+P_{s, t}^{\text {dis }}\right)+c^{\text {shed }} P_{l, t}^{\text {shed }}
\end{aligned}
$$

where $c^{\text {inv }}, c^{\text {op }}$ and $c^{\text {shed }}$ are constants and $\alpha^{\text {ann }}$ is the annuity factor computed by $\frac{r}{1-1 /(1+r)^{l}}$ with $r$ and $l$ denoting the rate of interest and the lifetime of the candidate unit, respectively.

Thus, the optimization problem can be formulated as

$$
\begin{aligned}
& \min C^{\mathrm{inv}}+\alpha^{\mathrm{d}} \sum_{t}^{T} C_{t}^{\mathrm{op}} \\
& \text { s.t. Constraints (1)-(15) }
\end{aligned}
$$


where $T$ is the total number of hours over all selected days and $\alpha^{\mathrm{d}}$ is equal to the total number of days of the examined year divided by the number of considered representative days.

\section{B. Uncertainty modelling using DRO}

In this subsection, the way to incorporate the uncertainties of wind, PV and demand forecasts into the model using DRO is illustrated. We first rewrite problem (16) in the following compact form:

$$
\begin{array}{ll}
\min & C^{\text {inv }}(\boldsymbol{x})+\phi(\boldsymbol{x}, \boldsymbol{\delta}) \\
\text { s.t. } & \boldsymbol{x} \in \boldsymbol{X}_{\boldsymbol{f}}
\end{array}
$$

where set $\boldsymbol{X}_{\boldsymbol{f}}$ is the feasibility region of the first-stage investment decision $\boldsymbol{x}=\left\{x_{k}^{\mathrm{inv}}, k \in K\right\}$ defined by (1). The first-stage investment $\operatorname{cost} C^{\text {inv }}(\boldsymbol{x})$ is a linear function of $\boldsymbol{x}$, whereas the second-stage cost expressed by $\phi(\boldsymbol{x}, \boldsymbol{\delta})$ is a function of both the first-stage decision $x$ and the realization of the random vector $\boldsymbol{\delta}=\left\{\boldsymbol{\delta}^{\mathrm{l}}, \boldsymbol{\delta}^{\mathrm{p}}, \boldsymbol{\delta}^{\mathrm{w}}\right\}$ with $\{l, p, w\}$ representing load, PV and wind respectively. As the DRO model considers the distribution uncertainty of the random variables, the second-stage problem aims to optimize the worst-case expectation over a family of distributions I within an ambiguity set $\mathbf{F}$, i.e.

$$
\begin{aligned}
\phi(\boldsymbol{x}, \boldsymbol{\delta})= & \min _{y} \sup _{\mathbf{I} \in \mathbf{F}} \mathbf{E}_{\mathbf{I}}\left\{\alpha^{\mathrm{d}} \sum_{t}^{T} C_{t}^{\mathrm{op}}\right\} \\
\text { s.t. } & \boldsymbol{A}(\boldsymbol{\delta})+\boldsymbol{B} \boldsymbol{y} \leq \boldsymbol{D}(\boldsymbol{\delta})
\end{aligned}
$$

where (18b) corresponds to (2)-(15), with $\boldsymbol{A}(\boldsymbol{\delta})$ and $\boldsymbol{D}(\boldsymbol{\delta})$ being affine functions of uncertainty $\delta$. Second-stage decisions are $\boldsymbol{y}=\left\{\boldsymbol{P}_{\boldsymbol{g}}, \boldsymbol{P}_{\boldsymbol{v}}, \boldsymbol{P}_{s}^{\mathrm{dis}}, \boldsymbol{P}_{s}^{\mathrm{ch}}, \boldsymbol{E}_{s}, \boldsymbol{P}_{l}^{\mathrm{sh}}, \boldsymbol{P}_{l}^{\text {shed }}\right\}$.

a) Modelling Ambiguity Set: The ambiguity set $\mathbf{F}$ is used to describe the possible distributions of uncertainty $\delta$ with their common statistical information. As the tractability of the problem is highly affected by the ambiguity set, we consider a SOC representable ambiguity set in this paper:

$$
\mathbf{F}=\left\{\begin{array}{ll} 
& E_{I}\left[\delta_{t}^{i}\right]=0 \\
\mathbf{I}: & E_{I}\left[\mid \delta_{t}^{i}\right] \leq \gamma_{1, t}^{i} \\
& E_{I}\left[\left(\delta_{t}^{i}\right)^{2}\right] \leq \gamma_{2, t}^{i} \\
& \operatorname{Pr}(\boldsymbol{\delta} \in W)=1
\end{array}\right\}
$$

where $i \in\{l, p, w\}$. The four lines in the ambiguity set $\mathbf{F}$ ensure that: (i) the expectation of $\delta_{t}^{i}$ for each time point $t$ and uncertainty $i$ should be zero; (ii) the upper bound of the expected absolute deviation is set as $\gamma_{1, t}^{i}$; (iii) the maximum variance expectation is defined as $\gamma_{2, t}^{i}$ and (iv) all realizations of $\delta$ are within an uncertainty set $W$, which is defined by the lower and upper bounds of $\delta_{t}^{i}$ for time $t$, i.e.

$$
W=\left\{\delta_{t}^{i, \min } \leq \delta_{t}^{i} \leq \delta_{t}^{i, \max }\right\}
$$

However, the expectation terms over functions of uncertainty $\delta_{t}^{i}$ in the second and third lines may affect the tractability of the optimization problem. To solve this issue, a lifting theorem was proposed in [11] to ensure the computational tractability and also to allow a richer modelling range of the ambiguity set. Following the principle of this theorem, a set of auxiliary random variables $\boldsymbol{u}=\left\{\boldsymbol{u}_{1}, \boldsymbol{u}_{2}\right\}$ is introduced. Finally, the following lifted ambiguity set $\overline{\mathbf{F}}$ that has only linear expectation constraints and a corresponding SOC representable extended uncertainty set $\bar{W}$ are obtained:

$$
\begin{aligned}
& \overline{\mathbf{F}}=\left\{\begin{array}{ll} 
& \\
\mathbf{J}: & E_{J}\left[\delta_{t}^{i}\right]=0 \\
& E_{J}\left[u_{1, t}^{i}\right] \leq \gamma_{1, t}^{i} \\
& E_{J}\left[u_{2, t}^{i}\right] \leq \gamma_{2, t}^{i} \\
& \operatorname{Pr}\{(\boldsymbol{\delta}, \boldsymbol{u}) \in \bar{W}\}=1
\end{array}\right\} \\
& \bar{W}=\left\{\begin{array}{c}
\delta_{t}^{i, \min } \leq \delta_{t}^{i} \leq \delta_{t}^{i, \max } \\
\left|\delta_{t}^{i}\right| \leq u_{1, t}^{i} \\
\left(\delta_{t}^{i}\right)^{2} \leq u_{2, t}^{i} \\
u_{1, t}^{i} \leq u_{1, t}^{i, \max }=\max \left\{\delta_{t}^{i, m a x},-\delta_{t}^{i, \min }\right\} \\
u_{2, t}^{i} \leq u_{2, t}^{i, \max }=\max \left\{\left(\delta_{t}^{i, \text { max }}\right)^{2},\left(\delta_{t}^{i, \text { min }}\right)^{2}\right\}
\end{array}\right\}
\end{aligned}
$$

where the second and third lines in set $\overline{\mathbf{F}}$ are equivalent to the second and third constraints in $\mathbf{F}$ as $\left|\delta_{t}^{i}\right|$ and $\left(\delta_{t}^{i}\right)^{2}$ are bounded by the incorporated auxiliary variables $u_{1, t}^{i}$ and $u_{2, t}^{i}$ with the second and third equations in $\bar{W}$, respectively. The choice of the upper limits of $u_{1, t}^{i}$ and $u_{2, t}^{i}$, namely $u_{1, t}^{i, \max }$ and $u_{2, t}^{i, \max }$, determines the level of conservatism of the solution. Similar to [7], in this work we limit $u_{1, t}^{i, \max }$ and $u_{2, t}^{i, \max }$ to the worstcase, i.e. $\max \left\{\delta_{t}^{i, \max },-\delta_{t}^{i, \min }\right\}$ and $\max \left\{\left(\delta_{t}^{i, \max }\right)^{2},\left(\delta_{t}^{i, m i n}\right)^{2}\right\}$. These boundary values are constants that can be derived from historical data. Further information about how to choose the upper bounds of auxiliary variables can be found in [12].

b) Enhanced LDR Approximation: Unfortunately, problem (18) is in general intractable, as the recourse problem needs to be solved by looping over all outcomes of random parameters [13]. To guarantee the computational tractability of the optimization problem, LDRs are selected to solve the problem by approximating the second-stage decision using linear functions. As incorporating auxiliary variables into the linear decision rule can improve the approximation [14], all second-stage decisions are modeled as affine functions of both the forecast error $\boldsymbol{\delta}$ and the auxiliary variable $\boldsymbol{u}$ as follows:

$$
\begin{aligned}
& P_{g, t}(\boldsymbol{\delta}, \boldsymbol{u})=k_{g, t}^{0}+\boldsymbol{k}_{\boldsymbol{g}, \boldsymbol{t}}^{\mathbf{1}} \boldsymbol{\delta}_{\boldsymbol{t}}+\boldsymbol{k}_{g, t}^{\mathbf{2}} \boldsymbol{u}_{1, t}+\boldsymbol{k}_{g, t}^{\mathbf{3}} \boldsymbol{u}_{2, t} \\
& P_{v, t}(\boldsymbol{\delta}, \boldsymbol{u})=k_{v, t}^{0}+\boldsymbol{k}_{\boldsymbol{v}, t}^{\mathbf{1}} \boldsymbol{\delta}_{t}+\boldsymbol{k}_{\boldsymbol{v}, t}^{\mathbf{2}} \boldsymbol{u}_{1, t}+\boldsymbol{k}_{v, t}^{\mathbf{3}} \boldsymbol{u}_{2, t} \\
& P_{s, t}^{\mathrm{dis}}(\boldsymbol{\delta}, \boldsymbol{u})=k_{\mathrm{dis}, t}^{0}+\boldsymbol{k}_{\mathrm{dis}, t}^{1} \boldsymbol{\delta}_{t}+\boldsymbol{k}_{\mathrm{dis}, t}^{2} \boldsymbol{u}_{1, t}+\boldsymbol{k}_{\mathrm{dis}, t}^{\mathbf{3}} \boldsymbol{u}_{2, t} \\
& P_{s, t}^{\mathrm{ch}}(\boldsymbol{\delta}, \boldsymbol{u})=k_{\mathrm{ch}, t}^{0}+\boldsymbol{k}_{\mathrm{ch}, t}^{\mathbf{1}} \boldsymbol{\delta}_{\boldsymbol{t}}+\boldsymbol{k}_{\mathrm{ch}, t}^{2} \boldsymbol{u}_{1, t}+\boldsymbol{k}_{\mathrm{ch}, \boldsymbol{t}}^{\mathbf{3}} \boldsymbol{u}_{2, t} \\
& E_{s, t}(\boldsymbol{\delta}, \boldsymbol{u})=k_{s, t}^{0}+\boldsymbol{k}_{s, t}^{\mathbf{1}} \boldsymbol{\delta}_{\boldsymbol{t}}+\boldsymbol{k}_{s, t}^{\mathbf{2}} \boldsymbol{u}_{1, t}+\boldsymbol{k}_{s, t}^{\mathbf{3}} \boldsymbol{u}_{2, t} \\
& P_{l, t}^{\mathrm{sh}}(\boldsymbol{\delta}, \boldsymbol{u})=k_{\mathrm{sh}, t}^{0}+\boldsymbol{k}_{\mathrm{sh}, \boldsymbol{t}}^{\mathbf{1}} \boldsymbol{\delta}_{\boldsymbol{t}}+\boldsymbol{k}_{\mathrm{sh}, \boldsymbol{t}}^{\mathbf{2}} \boldsymbol{u}_{1, t}+\boldsymbol{k}_{\mathrm{sh}, \boldsymbol{t}}^{\mathbf{3}} \boldsymbol{u}_{2, t} \\
& P_{l, t}^{\text {shed }}(\boldsymbol{\delta}, \boldsymbol{u})=k_{\text {shed }, t}^{0}+\boldsymbol{k}_{\text {shed }, \boldsymbol{t}}^{\mathbf{1}} \boldsymbol{\delta}_{\boldsymbol{t}}+\boldsymbol{k}_{\text {shed }, \boldsymbol{t}}^{\mathbf{2}} \boldsymbol{u}_{1, t}+\boldsymbol{k}_{\text {shed }, \boldsymbol{t}}^{\mathbf{3}} \boldsymbol{u}_{2, t}
\end{aligned}
$$

where $k_{j, t}^{0} \in \mathbb{R}, k_{j, t}^{1} \in \mathbb{R}^{I_{1}} k_{j, t}^{2} \in \mathbb{R}^{I_{1}}$ and $k_{j, t}^{3} \in \mathbb{R}^{I_{1}}$ are coefficient parameters with $j \in\{g, v, d i s, c h, s$, sh, shed $\}$ and $I_{1}$ representing the number of uncertainties. As the LDR limits the recourse decision $\boldsymbol{y}$ to be affinely dependent on $\boldsymbol{\delta}$ and $\boldsymbol{u}$, the second-stage problem now can be solved by optimizing the coefficients $k_{j, t}^{0,1,2,3}$ instead of evaluating all possible values of $\boldsymbol{y}$. Substituting (19) into constraints (18) we have:

$$
\begin{aligned}
\phi(\boldsymbol{x}, \boldsymbol{\delta})= & \min _{\substack{k_{j, t}^{0,1,2,3} \\
\text { s.t. }}} \sup _{\mathbf{J} \in \overline{\mathbf{F}}} \mathbf{E}_{\mathbf{J}}\left\{\alpha^{\mathrm{d}} \sum_{t}^{T} C_{t}^{\mathrm{op}}(\boldsymbol{\delta}, \boldsymbol{u})\right\} \boldsymbol{B} \boldsymbol{y}(\boldsymbol{\delta}, \boldsymbol{u}) \leq \boldsymbol{D}(\boldsymbol{\delta})
\end{aligned}
$$

c) Reformulation as a robust optimization problem: The introduced lifted ambiguity set $\overline{\mathbf{F}}$ and linear decision rules allow reformulating the inner maximization problem of (20) as the following continuous linear programming problem: 


$$
\begin{array}{ll} 
& \sup _{\mathbf{J} \in \overline{\mathbf{F}}} \mathbf{E}_{\mathbf{J}}\left\{\alpha^{\mathrm{d}} \sum_{t}^{T} C_{t}^{\mathrm{op}}(\boldsymbol{\delta}, \boldsymbol{u})\right\} \\
= & \sup \int_{\bar{W}} \alpha^{\mathrm{d}} \sum_{t}^{T} C_{t}^{\mathrm{op}}(\boldsymbol{\delta}, \boldsymbol{u}) d f(\boldsymbol{\delta}, \boldsymbol{u}) \\
\text { s.t. } & \int_{\bar{W}} \boldsymbol{\delta} d f(\boldsymbol{\delta}, \boldsymbol{u})=0 \quad: \boldsymbol{\rho} \\
& \int_{\bar{W}} \boldsymbol{u} d f(\boldsymbol{\delta}, \boldsymbol{u}) \leq \boldsymbol{\gamma} \\
& \int_{\bar{W}} d f(\boldsymbol{\delta}, \boldsymbol{u})=\mathbf{1} \quad: \eta \\
& f(\boldsymbol{\delta}, \boldsymbol{u}) \geq 0 \quad \forall(\boldsymbol{\delta}, \boldsymbol{u}) \in \bar{W} \\
& \boldsymbol{A}(\boldsymbol{\delta})+\boldsymbol{B} \boldsymbol{y}(\boldsymbol{\delta}, \boldsymbol{u}) \leq \boldsymbol{D}(\boldsymbol{\delta})
\end{array}
$$

denoting the probability density function as $f(\boldsymbol{\delta}, \boldsymbol{u})$. Variables $\boldsymbol{\rho}, \boldsymbol{\beta}$ and $\boldsymbol{\eta}$ indicate the dual variables of equations (21b)(21d), which are equivalent to formulations of the constraints in set $\overline{\mathbf{F}}$. According to the Lagrange duality theory in [15], the optimal value of its dual problem exists only if the constraint $\eta+\boldsymbol{\rho} \boldsymbol{\delta}+\boldsymbol{\beta} \boldsymbol{u} \geq \alpha^{\mathrm{d}} \sum_{t} C_{t}^{\mathrm{op}}(\boldsymbol{\delta}, \boldsymbol{u})$ is satisfied. Therefore, the dual problem of (21a)-(21f) is formulated as:

$$
\begin{array}{ll}
\min & \eta+\boldsymbol{\gamma} \boldsymbol{\beta} \\
\text { s.t. } & \boldsymbol{\beta} \geq 0 \\
& \eta+\boldsymbol{\rho} \boldsymbol{\delta}+\boldsymbol{\beta} \boldsymbol{u} \geq \alpha^{\mathrm{d}} \sum_{t} C_{t}^{\mathrm{op}}(\boldsymbol{\delta}, \boldsymbol{u}) \\
& \boldsymbol{A}(\boldsymbol{\delta})+\boldsymbol{B} \boldsymbol{y}(\boldsymbol{\delta}, \boldsymbol{u}) \leq \boldsymbol{D}(\boldsymbol{\delta})
\end{array}
$$

Taking the first-stage constraint and objective into consideration, the complete two-stage optimization problem is:

$$
\begin{array}{ll}
\min & C^{\mathrm{inv}}(\boldsymbol{x})+\eta+\boldsymbol{\gamma} \boldsymbol{\beta} \\
\text { s.t. } & \boldsymbol{\beta} \geq 0 \\
& \eta+\boldsymbol{\rho} \boldsymbol{\delta}+\boldsymbol{\beta} \boldsymbol{u} \geq \alpha^{\mathrm{d}} \sum_{t} C_{t}^{\mathrm{op}}(\boldsymbol{\delta}, \boldsymbol{u}) \\
& \boldsymbol{A}(\boldsymbol{\delta})+\boldsymbol{B} \boldsymbol{y}(\boldsymbol{\delta}, \boldsymbol{u}) \leq \boldsymbol{D}(\boldsymbol{\delta}) \\
& \boldsymbol{x} \in \boldsymbol{X}_{\boldsymbol{f}}
\end{array}
$$

where $\boldsymbol{\delta}$ and $\boldsymbol{u}$ in problem (23) should also fulfill the constraints in the support set $\bar{W}$. Thus, the way to incorporate these constraints into the $\boldsymbol{\delta}$ - and $\boldsymbol{u}$-related constraints in (23), i.e. (23c)-(23d), is described as follows. Taking constraint (23c) as an example, it is fulfilled only if the worst-case $\min \left\{\eta+\boldsymbol{\rho} \boldsymbol{\delta}+\boldsymbol{\beta} \boldsymbol{u}-\alpha^{\mathrm{d}} \sum_{t} C_{t}^{\mathrm{op}}(\boldsymbol{\delta}, \boldsymbol{u})\right\} \geq 0$ is fulfilled. Therefore, an optimization problem that is equivalent to constraint (23c) and also subject to constraints in set $\bar{W}$ is formulated:

$$
\begin{aligned}
& \min _{\mathbf{J} \in \overline{\mathbf{F}}}\left\{\eta+\boldsymbol{\rho} \boldsymbol{\delta}+\boldsymbol{\beta} \boldsymbol{u}-\alpha^{\mathrm{d}} \sum_{t} C_{t}^{\mathrm{op}}(\boldsymbol{\delta}, \boldsymbol{u})\right\} \geq 0 \\
& \text { s.t. }\left\|-\boldsymbol{\delta}+\boldsymbol{\delta}^{\mathrm{min}}+1\right\| \leq \boldsymbol{\delta}-\boldsymbol{\delta}^{\mathrm{min}}+1 \quad: \boldsymbol{\pi}^{\mathbf{1}}, \boldsymbol{\zeta}^{\mathbf{1}} \\
& \|\delta\| \leq \delta^{\max } \quad: \pi^{2}, \zeta^{2} \\
& \|\delta\| \leq u_{1} \quad: \pi^{3}, \zeta^{3} \\
& \left\|\boldsymbol{u}_{1}\right\| \leq \boldsymbol{u}_{1}^{\max } \quad: \boldsymbol{\pi}^{4}, \boldsymbol{\zeta}^{4} \\
& \left\|\left(\begin{array}{c}
\frac{1-\boldsymbol{u}_{2}}{2} \\
\boldsymbol{\delta}
\end{array}\right)\right\| \leq \frac{\boldsymbol{u}_{2}+1}{2} \quad: \boldsymbol{\pi}^{\mathbf{5}}, \boldsymbol{\zeta}^{\mathbf{5}} \\
& \left\|\boldsymbol{u}_{2}\right\| \leq \boldsymbol{u}_{\mathbf{2}}^{\max } \quad: \boldsymbol{\pi}^{6}, \boldsymbol{\zeta}^{6}
\end{aligned}
$$

where constraints in set $\bar{W}$ are rewritten in SOC forms with (25a)-(25b) and (25c)-(25e) corresponding to the first and the second to fifth lines in set $\bar{W} \cdot \boldsymbol{\pi}^{\mathbf{1} \boldsymbol{6}}$ and $\zeta^{\mathbf{1} \sim 6}$ are the corresponding dual variables.

Based on the Lagrange duality theory proposed in [15] and [16], (24) and (25) are transformed into the following equivalent constraints:

$$
\begin{aligned}
& \eta-\alpha^{\mathrm{d}} \sum_{t}\left[\sum_{l \in\{V, g\}} c_{l}^{\mathrm{op}} k_{l, t}^{0}+\left(c_{s}^{\mathrm{ch}} k_{\mathrm{ch}, t}^{0}+c_{s}^{\mathrm{dis}} k_{\mathrm{dis}, t}^{0}\right)\right. \\
& -\sum_{i}\left(\delta_{t}^{\mathrm{i}, \mathrm{min} 1} \pi_{t}^{i, 1}+\delta_{t}^{i, \min 2} \zeta_{t}^{i, 1}+\delta_{t}^{i, \max } \zeta_{t}^{i, 2}+u_{1, t}^{i, \mathrm{max}} \zeta_{t}^{i, 4}\right. \\
& \left.\left.\quad+[1 / 2,0] \pi_{t}^{i, 5}+\zeta_{t}^{i, 5} / 2+u_{2, t}^{i, \max } \zeta_{t}^{i, 6}\right)\right] \geq 0 \\
& \sum_{t}\left(-\pi_{t}^{i, 1}+\zeta_{t}^{i, 1}+\pi_{t}^{i, 2}-\pi_{t}^{i, 3}+[0,1] \pi_{t}^{i, 5}\right) \\
& =\sum_{t}\left[\rho_{t}^{i}+\alpha^{\mathrm{d}}\left(\sum_{l \in\{V, g\}} c_{l}^{\mathrm{op}} k_{l, t}^{i, 1}+\left(c_{s}^{\mathrm{ch}} k_{\mathrm{ch}, t}^{i, 1}+c_{s}^{\mathrm{dis}} k_{\mathrm{dis}, t}^{i, 1}\right)\right)\right] \\
& \sum_{t}\left(\zeta_{t}^{i, 3}+\pi_{t}^{i, 4}\right) \\
& =\sum_{t}\left[\beta_{t}^{i, 1}+\alpha^{\mathrm{d}}\left(\sum_{l \in\{V, g\}} c_{l}^{\mathrm{op}} k_{l, t}^{i, 2}+\left(c_{s}^{\mathrm{ch}} k_{\mathrm{ch}, t}^{i, 2}+c_{s}^{\mathrm{dis}} k_{\mathrm{dis}, t}^{i, 2}\right)\right)\right] \\
& \sum_{t}\left([-1 / 2,0] \pi_{t}^{i, 5}+\zeta_{t}^{i, 5} / 2+\pi_{t}^{i, 6}\right) \\
& =\sum_{t}\left[\beta_{t}^{i, 2}+\alpha^{\mathrm{d}}\left(\sum_{l \in\{V, g\}} c_{l}^{\mathrm{op}} k_{l, t}^{i, 3}+\left(c_{s}^{\mathrm{ch}} k_{\mathrm{ch}, t}^{i, 3}+c_{s}^{\mathrm{dis}} k_{\mathrm{dis}, t}^{i, 3}\right)\right)\right] \\
& \left\|\pi_{t}^{i, m}\right\| \leq \zeta_{t}^{i, m}, 0 \leq \zeta_{t}^{i, m} m=1,2, \ldots, 6 ; i=1,2, \ldots, I_{1}
\end{aligned}
$$

Consequently, constraint (23c) can be replaced by (26a)-(26e). Constraint (23d), corresponding to (2)-(15), can be rewritten following a similar process as that for constraint (23c).

Finally, the overall optimization problem can be written as:

$$
\begin{array}{ll}
\min & C^{\text {inv }}(\boldsymbol{x})+\eta+\boldsymbol{\gamma} \boldsymbol{\beta} \\
\text { s.t. } & \boldsymbol{\beta} \geq 0 \\
& \text { Constraints (26a)-(26e) } \\
& \text { Reformulated constraints equivalent to (2)-(15) in (23d) } \\
& \boldsymbol{x} \in \boldsymbol{X}_{\boldsymbol{f}}
\end{array}
$$

\section{Alternative uncertainty modelling methods}

As we intend to compare the results of DRO against that of $\mathrm{SO}$ and $\mathrm{RO}$ in this paper, formulations using $\mathrm{SO}$ and $\mathrm{RO}$ are defined as follows.

1) SO Formulation: In traditional $\mathrm{SO}$, it is assumed that the random variable $\delta$ can be modeled by a known probability distribution I. To solve the problem in a tractable manner, a set of scenarios are drawn from the distribution to describe the randomness of the uncertain variable and the objective is to minimize the first-stage cost plus the expected second-stage cost over all scenarios, i.e.

$$
\begin{aligned}
& \min C^{\mathrm{inv}}+\sum_{\omega} \operatorname{prob}_{\omega} \alpha^{\mathrm{d}} \sum_{t}^{T} C_{t, \omega}^{\mathrm{op}} \\
& \text { s.t. Constraints (1)-(15) for each scenario } \omega
\end{aligned}
$$

where $\operatorname{prob}_{\omega}$ is the realization probability of each scenario $\omega$. 
TABLE I: Parameters for candidate units

\begin{tabular}{|c|c|c|c|c|}
\hline Category & Type & $\begin{array}{c}\text { Investment cost } \\
\text { (kUSD/kW) }\end{array}$ & $\begin{array}{c}\text { Generation cost } \\
\text { (cents/kWh) }\end{array}$ & $\begin{array}{c}\text { Lifetime } \\
\text { (years) }\end{array}$ \\
\hline $\begin{array}{c}\text { Variable } \\
\text { generators }\end{array}$ & PV & 1.3 & 0 & 35 \\
\cline { 2 - 5 } & Wind & 0.8 & 0 & 25 \\
\hline $\begin{array}{c}\text { Dispatchable } \\
\text { generators }\end{array}$ & CHP & 3.7 & 1.7 & 20 \\
\hline $\begin{array}{c}\text { Storage } \\
\text { units }\end{array}$ & Battery & 0.2 & 0 & 15 \\
\hline
\end{tabular}

2) RO Formulation: In RO, $\delta$ is described by an uncertainty set $\mathbf{U}$, and the objective is to optimize the first-stage cost plus the worst-case optimal second-stage cost, i.e.

$$
\min C^{\text {inv }}+\sup _{\delta \in \mathbf{U}}\left\{\alpha^{\mathrm{d}} \sum_{t}^{T} C_{t}^{\text {op }}\right\}
$$

s.t. Constraints (1)-(15)

\section{CAse Study}

\section{A. Data}

For the simulation, we select 2050 as the examined year. Considering the computational burden, four days are selected to represent the variations in supply and demand over the year. Table I summarizes the parameters used in the case study. For the output of wind and PV units, we use historical hourly output data of four days in 2016 generated by Renewables.ninja [17] (location: Zurich). The conversion efficiency of the storage unit is assumed to be $92.5 \%$. The power to energy ratio is $50 \%$. The initial and final storage levels of batteries are set to $50 \%$. The load shedding cost is $300 \$ / \mathrm{MWh}$. Scaled Swiss historical demand with hourly peak load of 161.8 MW and minimum hourly load of 103.7 MW are used as estimated load profile that should be covered by DERs. The maximum load shift is set to be $10 \%$ of the forecast. The rate of interest is assumed to be $5 \%$. To build the DRO model, we randomly generate a simulation set (set 1) of 10,000 Gaussian distributed samples of $\left\{\delta^{w}, \delta^{p}, \delta^{l}\right\}$ with zero mean, and the corresponding standard deviation is 0.2 times the forecasted values for wind and PV outputs, and 0.1 times the load forecasted values. The values of the statistical parameters are calculated using simulation set 1 .

\section{B. Results}

We first validate the effectiveness of the proposed model by analyzing a base case. Then we investigate the effects of considering different statistical constraints in the ambiguity set and the impacts of the values of the statistical parameter. In the end, the comparison between RO, SO and DRO is conducted.

1) Base Case Analysis: In the base case scenario, it is assumed that all statistical information related to the expectation, the mean absolute deviation and the variance of forecast errors' distribution is used to build the ambiguity set. In this case, the aggregator invests in 130.9 MW dispatchable generation, 91.2 MW wind and 7.0 MWh battery. In general, more is invested than required as uncertainties regarding demand forecast are considered. Wind units are invested as the cheapest among all generation units and batteries are invested to reduce the variations caused by wind generations. Even though the conventional unit is not cost-competitive against PV and wind units, it does not suffer from forecast uncertainty
TABLE II: Impact of different statistical constraints

\begin{tabular}{|c|c|c|c|c|c|}
\hline $\begin{array}{c}\text { Statistical } \\
\text { data considered }\end{array}$ & $\begin{array}{c}\text { Dispatchable } \\
\text { Unit (MW) }\end{array}$ & $\begin{array}{c}\text { Wind } \\
\text { (MW) }\end{array}$ & $\begin{array}{c}\text { Storage } \\
\text { (MWh) }\end{array}$ & $\begin{array}{c}\text { Investment } \\
\text { costs } \\
\text { (mUSD) }\end{array}$ & $\begin{array}{c}\text { Costs } \\
\text { (mUSD) }\end{array}$ \\
\hline DRO1: Expectation & 161.4 & 115.3 & 0.6 & 54.5 & 71.5 \\
\hline $\begin{array}{c}\text { DRO2: Expectation, } \\
\text { absolute deviation }\end{array}$ & 121.7 & 162.2 & 15.7 & 45.7 & 68.1 \\
\hline $\begin{array}{c}\text { DRO3: Expectation, } \\
\text { absolute deviation, } \\
\text { variance }\end{array}$ & 130.9 & 91.2 & 7.0 & 44.2 & 66.1 \\
\hline
\end{tabular}

TABLE III: Impact of demand forecast errors' variances

\begin{tabular}{|c|c|c|c|c|}
\hline $\begin{array}{c}\text { Standard } \\
\text { deviation of } \delta^{l}\end{array}$ & $\begin{array}{c}\text { Dispatchable } \\
\text { Unit }(\mathrm{MW})\end{array}$ & $\begin{array}{c}\text { Wind } \\
(\mathrm{MW})\end{array}$ & $\begin{array}{c}\text { Storage } \\
(\mathrm{MWh})\end{array}$ & $\begin{array}{c}\text { Costs } \\
(\mathrm{mUSD})\end{array}$ \\
\hline $0.05 P_{l}$ & 126.0 & 84.1 & 17.4 & 62.3 \\
\hline $0.1 P_{l}$ & 130.9 & 91.2 & 7.0 & 66.1 \\
\hline $0.15 P_{l}$ & 136.3 & 93.4 & 0 & 70.3 \\
\hline
\end{tabular}

and is dispatchable. Therefore, it is invested in to guarantee the energy supply during low variable generation hours.

2) Effects of statistical constraints in ambiguity set: In this part, case studies are conducted to demonstrate how investment decisions and the objective are affected by considering different types of statistical constraints and by changing values of statistical information in the ambiguity set.

Table II shows the results of the proposed DRO model with different types of statistical data included in the ambiguity set to capture the distribution of forecast errors. It shows that investment and total costs decrease when more statistical constraints are considered as the worst-case distribution will be improved when more statistical information is considered. Compared to DRO1, DRO2 introduced the variability of $\boldsymbol{\delta}$ by including the absolute deviation and improves the expected worst-case wind generation. Thus, more wind is invested to replace dispatchable units in serving the load as it is cheaper, storage investment is increased to cover the variance of net demand. DRO3 limited the variability of $\delta$ by including a variance limit, therefore both wind and storage investments decrease as the expected worst-case wind and demand distributions are further improved and the variability decreased.

To analyze the effects of different values of statistical parameters, we then conduct simulations with different standard deviations (SD) of demand forecast error. As shown in Table III, the total costs increase as the forecast errors' variance increases. When the SD of demand forecast error increases, more is invested in dispatchable generators and wind while less in batteries. This is because the peak demand increases with the increased standard deviation, thus more wind and dispatchable generation are invested as the wind unit is cheap and its output pattern is abundant while dispatchable units can provide both power and flexibility so as to cover the variance of the demand.

3) DRO vs. SO vs. RO: To compare the performance of SO, RO and DRO, we carry out the following simulations:

(a) Generate another set of scenarios as test set (i.e. set 2) using different distributions, namely Gaussian, uniform and Weibull distribution. The assumptions of expectations, variance and the number of samples of set 2 are the same as that of set 1 . Set 1 serves as the set to optimize investment decisions and set 2 serves as the out-of-sample test set to validate the optimality of the methods.

(b) With the information of set 1 , we optimize the first-stage investment decisions using $\mathrm{SO}, \mathrm{RO}$ and DRO. 
TABLE V: Impact of disturbances on different models

\begin{tabular}{|c|c|c|c|c|c|c|c|c|c|c|c|c|c|c|c|}
\hline \multicolumn{16}{|c|}{ Total out-of-sample expected costs (mUSD) } \\
\hline & \multicolumn{3}{|c|}{ RO } & \multicolumn{3}{|c|}{ DRO1 } & \multicolumn{3}{|c|}{ DRO2 } & \multicolumn{3}{|c|}{ DRO3 } & \multicolumn{3}{|c|}{ SO } \\
\hline & $\begin{array}{c}\text { load } \\
\text { shedding } \\
\text { cost }\end{array}$ & $\begin{array}{c}\text { residual } \\
\text { cost }\end{array}$ & $\begin{array}{l}\text { total } \\
\text { cost }\end{array}$ & $\begin{array}{c}\text { load } \\
\text { shedding } \\
\text { cost }\end{array}$ & $\begin{array}{c}\text { residual } \\
\text { cost }\end{array}$ & $\begin{array}{l}\text { total } \\
\text { cost }\end{array}$ & $\begin{array}{c}\text { load } \\
\text { shedding } \\
\text { cost }\end{array}$ & $\begin{array}{c}\text { residual } \\
\text { cost }\end{array}$ & $\begin{array}{l}\text { total } \\
\text { cost }\end{array}$ & $\begin{array}{c}\text { load } \\
\text { shedding } \\
\text { cost }\end{array}$ & $\begin{array}{c}\text { residual } \\
\text { cost }\end{array}$ & $\begin{array}{l}\text { total } \\
\text { cost }\end{array}$ & $\begin{array}{c}\text { load } \\
\text { shedding } \\
\text { cost }\end{array}$ & $\begin{array}{c}\text { residual } \\
\text { cost }\end{array}$ & $\begin{array}{l}\text { total } \\
\text { cost }\end{array}$ \\
\hline Case 1 & 0 & 78.0 & 78.0 & 0.3 & 71.3 & 71.6 & 4.6 & 61.2 & 65.8 & 4.4 & 61.3 & 65.7 & 2.1 & 59.8 & 61.9 \\
\hline Case 2 & 0 & 78.0 & 78.0 & 1.1 & 71.2 & 72.3 & 8.0 & 61.0 & 69.0 & 7.9 & 61.1 & 69.0 & 4.4 & 59.7 & 64.1 \\
\hline Case 3 & 0 & 78.0 & 78.0 & 2.6 & 71.2 & 73.8 & 12.1 & 60.8 & 72.9 & 12.1 & 60.9 & 73.0 & 7.6 & 59.6 & 67.1 \\
\hline Case 4 & 0 & 78.0 & 78.0 & 4.8 & 71.1 & 75.9 & 16.8 & 60.6 & 77.3 & 17.0 & 60.6 & 77.6 & 11.4 & 59.4 & 70.7 \\
\hline
\end{tabular}

TABLE IV: Comparison of different optimization methods

\begin{tabular}{|c|c|c|c|c|c|}
\hline \multicolumn{7}{|c|}{ Simulation results using set 1 } \\
\hline & RO & DRO1 & DRO2 & DRO3 & SO \\
\hline Total costs (mUSD) & 85.5 & 71.5 & 68.1 & 66.1 & 60.4 \\
\hline Computational time (s) & 8 & 11 & 10 & 1065 & 1385 \\
\hline \multicolumn{6}{|c|}{ Out-of-sample test results using set 2 } \\
\hline $\begin{array}{c}\text { Expected costs for } \\
\text { different scenarios (mUSD) }\end{array}$ & RO & DRO1 & DRO2 & DRO3 & SO \\
\hline Gaussian & 78.0 & 71.3 & 63.4 & 63.3 & 60.4 \\
\hline Uniform & 78.0 & 71.3 & 63.4 & 63.3 & 60.5 \\
\hline Weibull & 78.0 & 71.4 & 63.7 & 63.7 & 60.5 \\
\hline
\end{tabular}

(c) We apply the resulting optimal investment decisions achieved by SO, RO and DRO to the different distribution scenarios of set 2 and compare their performances and robustness by calculating the total expected costs.

We set the number of scenarios to 10,000 , as it is limited by the computational time of the SO model. Table IV shows the results of different optimization approaches. It is obvious that DRO performs better than RO, as it incorporates more uncertainty distribution information so as to provide less conservative results, however, the computational effort of using DRO is lower than that of SO. This effect is even more pronounced when more scenarios are considered. In contrast to SO, DRO is more conservative as its decisions are immune against a family of distributions. Comparing the results of DRO1-3, the performance generally improves as more statistical data is included.

To analyze the impacts of disturbances on the different models, we test the simulation results of different methods against four cases with standard deviation of Gaussian distributed scenarios increased by 0.05 times of the forecast in Case 1 (i.e. $\delta^{p}=0.25 P_{p}, \delta^{w}=0.25 P_{w}, \delta^{l}=0.15 P_{l}$ ) to 0.2 times of the forecast in Case 4 with a 0.05 increment. As shown in Table $\mathrm{V}$, the total expected costs are decomposed into load shedding costs and the residual costs. While DRO13 and SO models are sensitive to variance disturbances since their decisions are optimized assuming the known variance or specific distribution, the performance of RO is stable as the resulting decision is conservative by considering the worst case. Although SO shows the best results in all cases, the best performer among DRO1-3 shifts from DRO3 to DRO2 then to DRO1 with the variance increase. The reason is that as seen in Table $\mathrm{V}$ the increase in total costs is decided by the growth of the load shedding cost. Since DRO1 only includes expectation information into the ambiguity set, its investment decisions are the most conservative among DRO13 and its load shedding cost is thus least affected by the variance change. Therefore, with further increases in variance, DRO1 gradually outperforms the DRO2-3. Note that the load shedding cost highly depends on the value of $c^{\text {shed }}$, and it is high here since the considered distribution system is assumed isolated, i.e. the energy exchange with the transmission system is ignored.

\section{CONClusion}

A two-stage stochastic programming model using DRO to derive optimal investment and operations for a distribution system is presented in this paper. Numerical results show that compared to SO and RO, DRO is more appropriate in cases where large amount of historical data is available without knowing the specific probability distribution. Ambiguity sets must be constructed depending on the available statistical information and the related known certainty. For future research, chance-constrained ambiguity sets will be considered and a more extensive case study will be carried out.

\section{REFERENCES}

[1] P. Yang and A. Nehorai, "Joint optimization of hybrid energy storage and generation capacity with renewable energy," IEEE Transactions on Smart Grid, vol. 5, no. 4, pp. 1566-1574, 2014.

[2] W. Yao, C. Chung, F. Wen, M. Qin, and Y. Xue, "Scenario-based comprehensive expansion planning for distribution systems considering integration of plug-in electric vehicles," IEEE Transactions on Power Systems, vol. 31, no. 1, pp. 317-328, 2016.

[3] X. Shen, M. Shahidehpour, Y. Han, S. Zhu, and J. Zheng, "Expansion planning of active distribution networks with centralized and distributed energy storage systems," IEEE Transactions on Sustainable Energy, vol. 8, no. 1 , pp. 126-134, 2017.

[4] H. Wang and J. Huang, "Joint investment and operation of microgrid," IEEE Transactions on Smart Grid, vol. 8, no. 2, pp. 833-845, 2017.

[5] Z. Wang, B. Chen, J. Wang, J. Kim, and M. M. Begovic, "Robust optimization based optimal DG placement in microgrids," IEEE Transactions on Smart Grid, vol. 5, no. 5, pp. 2173-2182, 2014.

[6] P. M. Esfahani and D. Kuhn, "Data-driven distributionally robust optimization using the wasserstein metric: Performance guarantees and tractable reformulations," Mathematical Programming, pp. 1-52, 2017.

[7] P. Xiong, P. Jirutitijaroen, and C. Singh, "A distributionally robust optimization model for unit commitment considering uncertain wind power generation," IEEE Transactions on Power Systems, vol. 32, no. 1, pp. 39-49, 2017.

[8] W. Wei, F. Liu, and S. Mei, "Distributionally robust co-optimization of energy and reserve dispatch," IEEE Transactions on Sustainable Energy, vol. 7, no. 1, pp. 289-300, 2016.

[9] X. Han, E. G. Kardakos, and G. Hug, "Trading strategy for decentralized energy resources in sequential electricity markets: A swiss case study," in Innovative Smart Grid Technologies-Asia (ISGT-Asia), 2017.

[10] A. J. Conejo, M. Carrión, and J. M. Morales, Decision making under uncertainty in electricity markets. New York: Springer, 2010.

[11] W. Wiesemann, D. Kuhn, and M. Sim, "Distributionally robust convex optimization," Operations Research, vol. 62, no. 6, pp. 1358-1376, 2014.

[12] E. Delage and Y. Ye, "Distributionally robust optimization under moment uncertainty with application to data-driven problems," Operations research, vol. 58, no. 3, pp. 595-612, 2010.

[13] A. Ben-Tal, A. Goryashko, E. Guslitzer, and A. Nemirovski, "Adjustable robust solutions of uncertain linear programs," Mathematical Programming, vol. 99, no. 2, pp. 351-376, 2004.

[14] D. Bertsimas, M. Sim, and M. Zhang, "Adaptive distributionally robust optimization," Management Science, 2018.

[15] A. Shapiro, On duality theory of conic linear problems. Semi-Infinite Programming. Springer US, 2001.

[16] S. Boyd and L. Vandenberghe, Convex Optimization. Cambridge university press, 2004.

[17] I. Staffell and S. Pfenninger, "Using bias-corrected reanalysis to simulate current and future wind power output," Energy, vol. 114, pp. 1224-1239, 2016. 